\title{
Earth-like planets: science performance predictions for future nulling interferometry missions
}

\author{
D. Defrère*a ${ }^{*}$ O. Lay $^{b}$, R. den $\operatorname{Hartog}^{c}$, and O. Absil ${ }^{d}$ \\ ${ }^{a}$ Institut d'Astrophysique et de Géophysique, Université de Liège, 17 Allée du Six Août, \\ B-4000 Liège, Belgium \\ ${ }^{b}$ Jet Propulsion Laboratory, Pasadena, USA \\ ${ }^{c}$ Science Payloads and Advanced Concepts Office, ESA/ESTEC, The Netherlands \\ ${ }^{d}$ LAOG-UMR 5571, CNRS and Université Joseph Fourier, Grenoble, France
}

\begin{abstract}
With the launch of planet-transit missions such as CoRoT and Kepler, it is expected that Earth-sized planets orbiting distant stars will be detected soon. This milestone will open the path towards the definition of missions able to study the atmosphere of Earth-sized extrasolar planets, with the identification of bio-signatures as one of the main objectives. In that respect, both the European Space Agency (ESA) and the National Aeronautics and Space Administration (NASA) have identified nulling interferometry as one of the most promising techniques. Trying to minimize the cost and the technological risks while maximizing the scientific return, ESA and NASA recently converged towards a single mission architecture, the Emma X-array. In this paper, we present the expected science performance of this concept computed with two independent mission simulators. The impact of different observational parameters such as planet radius and exozodiacal cloud density is specifically addressed.
\end{abstract}

Keywords: Nulling interferometry, space-based mission, extrasolar planets, biosignatures.

\section{INTRODUCTION}

The study of extrasolar planets and the search for other habitable worlds are just beginning. Since the first planet discovered around another solar-type star in $1995,{ }^{1}$ nearly 300 extrasolar planets have been detected and many more are expected to be unveiled by ongoing or future search programmes. Most extrasolar planets detected so far have been evidenced from the ground by indirect techniques, which rely on observable effects induced by the planet on its parent star. Observing from the ground is however limited by the atmosphere and most detected extrasolar planets present a high mass (the median mass is about $1.5 \mathrm{M}_{\text {Jup }}$ ) and a short orbital period. Radial velocity measurements are currently limited to the detection of planets about 5 times as massive as the Earth in orbits around Sun like and low-mass stars ${ }^{2}$ while the transit method is limited to Neptunesized planets. ${ }^{3}$ Thanks to the very high precision photometry enabled by the stable space environment, first space-based dedicated missions (namely CoRoT and Kepler) are now expected to reveal Earth-sized extrasolar planets. Launched in December 2006, CoRoT (Convection Rotation and planetary Transits) has detected its first extrasolar planets ${ }^{4,5}$ and is expected to unveil about 100 transiting planets down to a size of $2 \mathrm{R}_{\oplus}$ around G0V stars and $1.1 \mathrm{R}_{\oplus}$ around M0V stars over its entire lifetime (at least for short orbital periods). ${ }^{6}$ Planned to be launched in 2009, Kepler will extend the survey to Earth-sized planets located in the habitable zone of about 100000 main sequence stars. ${ }^{7}$ After 4 years, Kepler should have discovered several hundred terrestrial planets with periods between one day and 400 days. After this initial reconnaissance by CoRot and Kepler, the Space Interferometry Mission (SIM PlanetQuest) will provide unambiguously the mass of Earth-sized extrasolar planets orbiting in the habitable zone of nearby stars by precise astrometric measurements.

With CoRoT, Kepler and SIM, we will have a large census of Earth-sized extrasolar planets and their occurrence frequency as a function of different stellar properties. However, even though the composition of the upper atmosphere of transiting extrasolar planets can be probed in favorable cases, ${ }^{8}$ none of these missions will detect directly the planetary photons which are required in order to study planetary atmospheres and eventually reveal

* E-mail: defrere@astro.ulg.ac.be, Telephone: 003243669713

Optical and Infrared Interferometry, edited by Markus Schöller, William C. Danchi, Françoise Delplancke Proc. of SPIE Vol. 7013, 701321, (2008) · 0277-786X/08/\$18 · doi: 10.1117/12.789393 
the signature of biological activity. Detecting the light from an Earth-like extrasolar planet is very challenging due to the high contrast $\left(\sim 10^{7}\right.$ in the mid-IR, $\sim 10^{10}$ in the visible) and the small angular separation $(\sim 0.5$ $\mu \mathrm{rad}$ for an Earth-Sun system located at $10 \mathrm{pc}$ ) between the planet and its host star. A technique that has been proposed to overcome these difficulties is nulling interferometry. ${ }^{9}$ The basic principle of nulling interferometry is to combine the beams coming from two telescopes in phase opposition so that a dark fringe appears on the line of sight, which strongly reduces the stellar emission. Considering the two-telescope interferometer initially proposed by Bracewell, the response on the plane of the sky is a serie of sinusoidal fringes, with angular spacing of $\lambda / b$. By adjusting the baseline length, the transmission of the off-axis planetary companion can then be maximised. However, even if the stellar emission is sufficiently reduced, it is generally not possible to detect Earth-like planets with a static array configuration, particularly due to the dominant exozodiacal emission. This is the reason why Bracewell proposed to rotate the interferometer so that the planet signal is temporally modulated by alternatively crossing high and low transmission regions, while the stellar signal and the background emission remain constant. The planetary signal can then be retrieved by synchronous demodulation. However, since the rotation of the array can not be implemented sufficiently fast, this observing scenario is highly vulnerable to low frequency drifts in the stray light, thermal emission, and detector gain. A number of interferometer configurations with more than two collectors have been proposed to perform faster modulation and overcome this problem by using phase chopping. ${ }^{10-12}$ The principle of phase chopping is to synthetise two different transmission maps with the same telescope array, by applying different phase shifts in the beam combination process. By quickly switching between the two different transmission maps, it is possible to modulate the planet signal without modulating the stellar, local zodiacal cloud, exozodiacal cloud, stray light, thermal, or detector gain contributions to the noise. Phase chopping can be implemented in various ways (e.g. inherent and internal modulation ${ }^{13}$ ), and are now an essential part of future space-based life-finding nulling interferometry missions such as ESA's DARWIN ${ }^{14}$ and NASA's Terrestrial Planet Finder (TPF). ${ }^{15}$ The DARWIN/TPF mission will be able to detect Earth-like planets and perform the spectroscopic analysis of their atmosphere at mid-infrared wavelengths $(6-20 \mu \mathrm{m})$. In addition to presenting the most advantageous star/planet contrast, this wavelength range comprises several spectral features relevant to the detection of biological activity $\left(\mathrm{CO}_{2}, \mathrm{H}_{2} \mathrm{O}, \mathrm{CH}_{4}, \mathrm{O}_{3}\right)$.

\section{EMMA CONFIGURATION}

Considerable efforts have been expended the past decade by both ESA and NASA to design a mission that provides the required scientific performance while minimizing cost and technical risks. After the investigation of several interferometer architectures, these efforts culminated in 2005-2006 with two parallel assessment studies of the DARwIN mission, carried out by EADS Astrium and Alcatel-Alenia Space. Two array architectures have been thoroughly investigated during these industrial studies: the four-telescope X-array and the Three-Telescope Nuller (TTN). ${ }^{16}$ These studies included the launch requirements, payload spacecraft, and the ground segment during which the actual mission science would be executed. Almost simultaneously, NASA/JPL initiated a similar study for the Terrestrial Planet Finder Interferometer (TPF-I). These efforts on both sides of the Atlantic have finally resulted in a convergence and consensus on mission architecture, the so-called non-coplanar or Emma-type $\mathrm{X}$-array (represented in Figure 1). The baseline design consists in four 2-m aperture collector spacecraft, flying in rectangular formation and feeding light to the beam combiner spacecraft located approximately $1200 \mathrm{~m}$ above the array. This arrangement makes available baselines up to $170 \mathrm{~m}$ for nulling measurements and up to $500 \mathrm{~m}$ for the general astrophysics programme (constructive imaging). Note that the size of the collecting apertures has not yet been fixed and will mainly depend on the final cost of the mission.

The optical layout of the $\mathrm{X}$-array configuration separates the nulling and imaging functions, the shortest baselines being used for nulling and the longest ones for imaging. This configuration has the advantage to allow optimal tuning of the shorter dimension of the array for starlight suppression while keeping a significantly longer dimension to fully resolve the planetary system. The $\mathrm{X}$-array design is also appropriate to implement various techniques for removing instability noise, which is generally the dominant noise contributor. The assessment studies settled on an imaging to nulling baseline ratio of 3:1, based on scientific and instrument design constraints. A somewhat larger ratio of 6:1 may improve performance by simplifying noise reduction in the post-processing of science images. ${ }^{18}$ Beam combination takes place on a series of optical benches arranged within the beam combiner spacecraft. The necessary optical processing includes: transfer optics and spacecraft metrology; correction and 


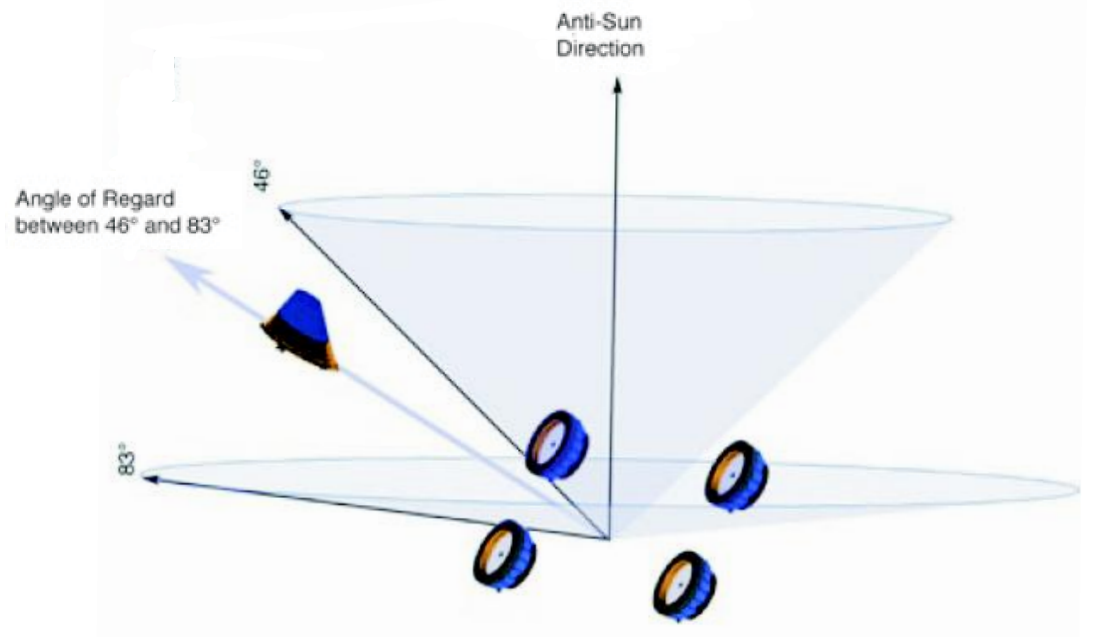

Figure 1. Representation of the DARwIN/TPF space interferometer in its baseline "Emma X-array" configuration. ${ }^{17}$ It presents 4 telescopes and a beam combiner spacecraft, deployed and observing at the Sun-Earth Lagrange point L2. At any given time, it can observe an annular region on the sky between $46^{\circ}$ and $83^{\circ}$ from the anti-Sun direction. During one Earth year, this annulus executes a complete circle, giving access to almost all regions of the celestial sphere.

modulation, including optical delay lines, tip-tilt, deformable mirrors; wavefront sensors and beam switching; spectral separation, if necessary, to feed the science photons into 2 separate channels; phase shifting, beam mixing; recombination, spectral dispersion and detection.

DARWIN/TPF is foreseen to be placed around the second Lagrange point (L2) by an Ariane 5 ECA vehicle. L2 is optimal to achieve passive cooling below $50 \mathrm{~K}$ of the collector and beam combiner spacecraft by means of sunshades. An additional refrigerator within the beam combiner spacecraft cools the detector assembly to below $10 \mathrm{~K}$. Due to the configuration of the array and the need for solar avoidance, the instantaneous sky access is limited to an annulus with inner and outer half-angles of $46^{\circ}$ and $83^{\circ}$ centred on the anti-sun vector (see Figure 1). ${ }^{19}$ This annulus transits the entire ecliptic circle during one year, giving access to almost the entire sky.

\section{THE SCIENCE SIMULATORS}

In order to provide realistic performance predictions, we use two mission simulators developed independently at ESA/ESTEC and NASA/JPL in the context of the DARWIN and TPF missions. The two simulators, DARWINsim $^{20}$ and the TPF count model, ${ }^{21}$ have the same basic purpose. For a given instrumental configuration and target catalogue, they assess the number of terrestrial planets that can be detected in the habitable zone of nearby main sequence stars and the number of possible follow-up spectroscopic observations during a nominal mission time. The duration of detection and spectroscopy phases can be adjusted to optimise the scientific return and is nominally set to 2 and 3 years respectively.

\subsection{Detection phase}

The starting point of the simulations is the target star catalogue. Given a specific interferometer architecture, the simulators first select the stars which are observable during one year from L2. For each of these observable stars, the basic calculation consists in an assessment of the required integration times to achieve a user-specified SNR for broad-band detection of a hypothetic Earth-like planet located inside the habitable zone. Since the location of the planet around the star is a priori unknown, each simulator considers its own strategy. 

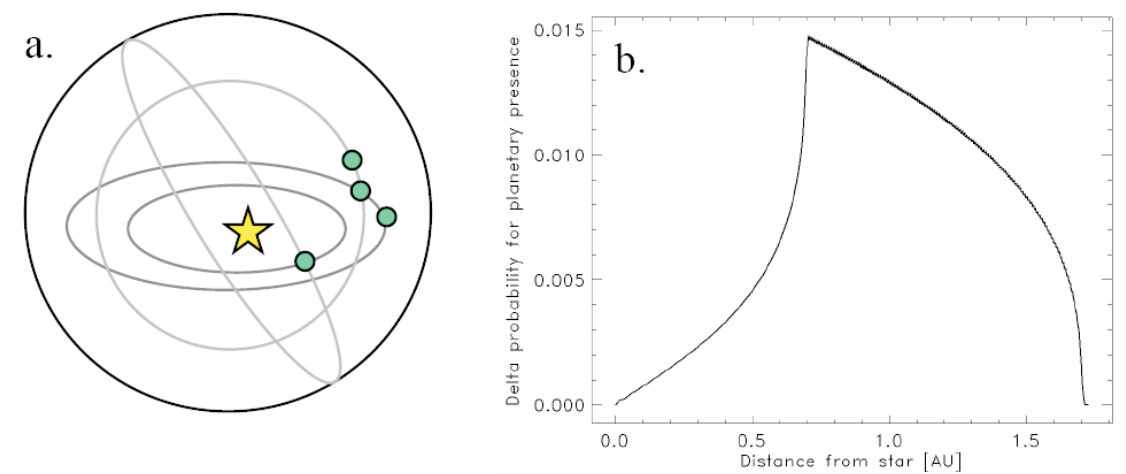

Figure 2. Left: a planet orbiting in the habitable zone of its host star can present various apparent distances, because the inclinations and phases of the orbits are a priori unknown. Right: probability distribution with respect to the apparent planet-star distance. ${ }^{23}$

\section{DARWInsim}

In DARwinsim, the integration time is computed from the requirement that it should ensure the detection of a planet for at least $90 \%$ of the possible locations in the habitable zone. Assuming planets uniformly distributed along the possible habitable orbits, this requires to compute the probability distribution for finding a planet at a certain angular distance from the star, as illustrated in Figure 2. For each planetary position, the maximum SNR is computed by the simultaneous optimisation of the baseline length and the wavelength range. The thermal flux of the habitable planet is assumed to be identical to that of Earth irrespective of the distance to the star. The flux of the exozodiacal clouds has been computed assuming a mean density 3 times larger than that in the solar system. Under the assumption that the exozodiacal emission is symmetric around the target star, it will be suppressed by the chopping process, and therefore only contributes to the shot noise. The noise sources included are the shot noise contributions from stellar leakage, local and exozodiacal clouds, and instrumental infrared background. Instability noise ${ }^{22}$ is also thoroughly implemented and is partly mitigated by phase chopping.

After the initial integration time assessment for detection, the targets are sorted by ascending integration time, removing from the list the targets for which the total integration time exceeds the total time during which they are visible from L2. Considering a slew time for re-targeting (nominally 6 hours) and an efficiency for the remaining observing time of $70 \%$, the sorted list is cut off at the moment when the cumulative integration time exceeds the nominal survey period. The resulting list defines the number of targets that can be surveyed during the detection phase.

\section{TPF count model}

In the TPF count model, the observing scenario is based on a similar approach for the TPF-C mission. Instead of searching all stars to a given level of completeness, the algorithm assesses the observability for a sample of 1000 planets uniformly distributed over the possible habitable orbits around each star of the catalogue. For each week of the mission, an optimization algorithm ${ }^{21}$ selects only the most productive target stars in order to maximize the number of planets found. At the end, the total number of planets found is divided by 1000 to obtain the average number of planets that would be detected if each star had only one planet in the habitable zone. Using this technique, the targets are not necessarily surveyed with the same level of completeness and it is likely that observing a larger number of stars at lower completeness is more efficient than observing fewer stars at high completeness.

The same noise sources as in DARWINsim are included and instability noise is assumed to be removed by the spectral fitting technique ${ }^{24}$ which induces a signal loss inherent to the process. At the time when the simulations were done, the target stars were selected from two different catalogues including 1132 stars $(114 \mathrm{~F}, 200 \mathrm{G}, 417 \mathrm{~K}$ and $401 \mathrm{M}$ stars) for DARWinsim and 1014 stars (5 A, $206 \mathrm{~F}, 375 \mathrm{G}, 416 \mathrm{~K}$ and $12 \mathrm{M}$ stars) for the TPF count model. These catalogues result from different selection criteria that were initially applied on both side of the 

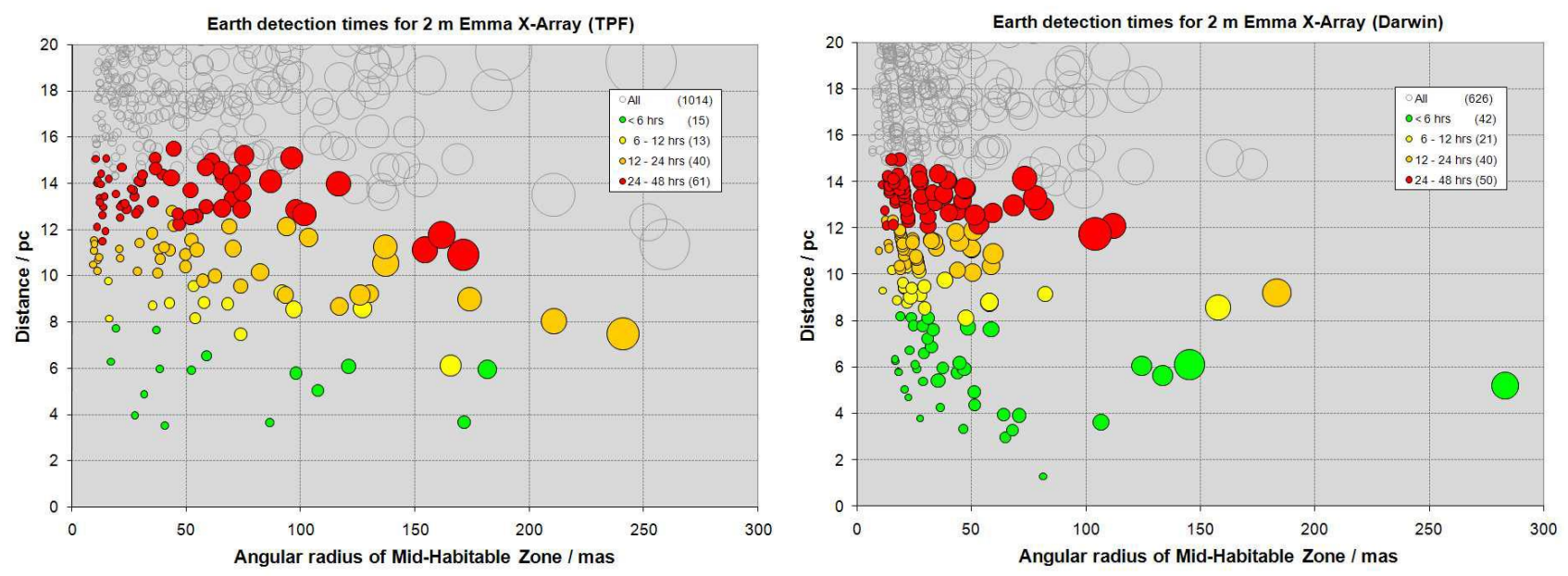

Figure 3. Integration times needed to detect with an SNR of 5 an Earth-like planet located in the middle of the habitable zone of candidate targets (represented as circles proportional to their intrinsic diameter) with the TPF count model (left) ${ }^{21}$ and DARWInsim (right). Green, yellow, orange and red circles show stars which require an integration time shorter than $6,12,24$ and 48 hours respectively.

Atlantic and are now converging towards a single target list. ${ }^{25}$ Considering an Earth-like planet in the middle of the habitable zone, Figure 3 shows the integration time required to achieved an SNR of 5 for each target star of the TPF catalogue with the TPF count model (left) and DARWIN catalogue with DARWINsim (right). Circle diameters are proportional to the intrinsic size of the star. The instability noise has been disabled in DARWINsim in order to produce a fair the comparison with the TPF count model. The two simulators show a very good agreement. For common target stars, the integration times computed with DARWINsim are in average $10 \%$ shorter than those computed with the TPF count model. This is partly due to planetary photons lost during the instability noise subtraction process taken into account in the TPF count model. Note that the integration time required to achieve a completeness level of $90 \%$ is in average about $30 \%$ longer than in the case where the planet is located in the middle of the habitable zone and at maximum elongation.

Since each simulator considers the detection of terrestrial planets at various apparent distances from the star, it is useful to extend the comparison of integration times for different angular offsets. This is illustrated by Figure 4 computed with the two simulators for a Sun-Earth system located at $10 \mathrm{pc}$ and showing the integration time with respect to the planet offset. In order to make a fair comparison, the instability noise has been disabled in the TPF count model to plot this figure. The two simulators give consistent integration times, suggesting that they should lead to similar results for the detection phase by using the same observing scenario.

\subsection{Spectroscopy phase}

The number of targets which can be characterised by low-resolution spectroscopy in a given time is computed similarly in the two simulators. The difference with the detection phase is that the integration times are computed for a given position in the habitable zone. The proper procedure would be to take into account all possible positions for the planet in a similar way to the detection phase but this would be far too time consuming. The strategy is then to consider only the most likely angular offset. Then, the total integration time is determined by the requirement to detect the absorption lines of $\mathrm{O}_{3}, \mathrm{CO}_{2}$ and $\mathrm{H}_{2} \mathrm{O}$ to a specified SNR. For the spectroscopy of $\mathrm{CO}_{2}$ and $\mathrm{O}_{3}$ (without $\mathrm{H}_{2} \mathrm{O}$ ), an SNR of 5 would actually be sufficient for a secure detection. ${ }^{26}$ Considering the spectroscopy of $\mathrm{H}_{2} \mathrm{O}$ is relatively more complex. Recent results suggest that, using a spectral resolution greater than 20, a SNR of 10 from 7.2 to $20 \mu \mathrm{m}$ would be sufficient for $\mathrm{H}_{2} \mathrm{O}, \mathrm{CO}_{2}$, and $\mathrm{O}_{3}$ spectroscopy (private communication with F. Selsis, L. Kaltenegger and J. Paillet). In particular, these results suggest that the $\mathrm{H}_{2} \mathrm{O}$ band located below $7.2 \mu \mathrm{m}$, which is much more constraining that the $H_{2} O$ band beyond $17.2 \mu \mathrm{m}$, could be discarded. 


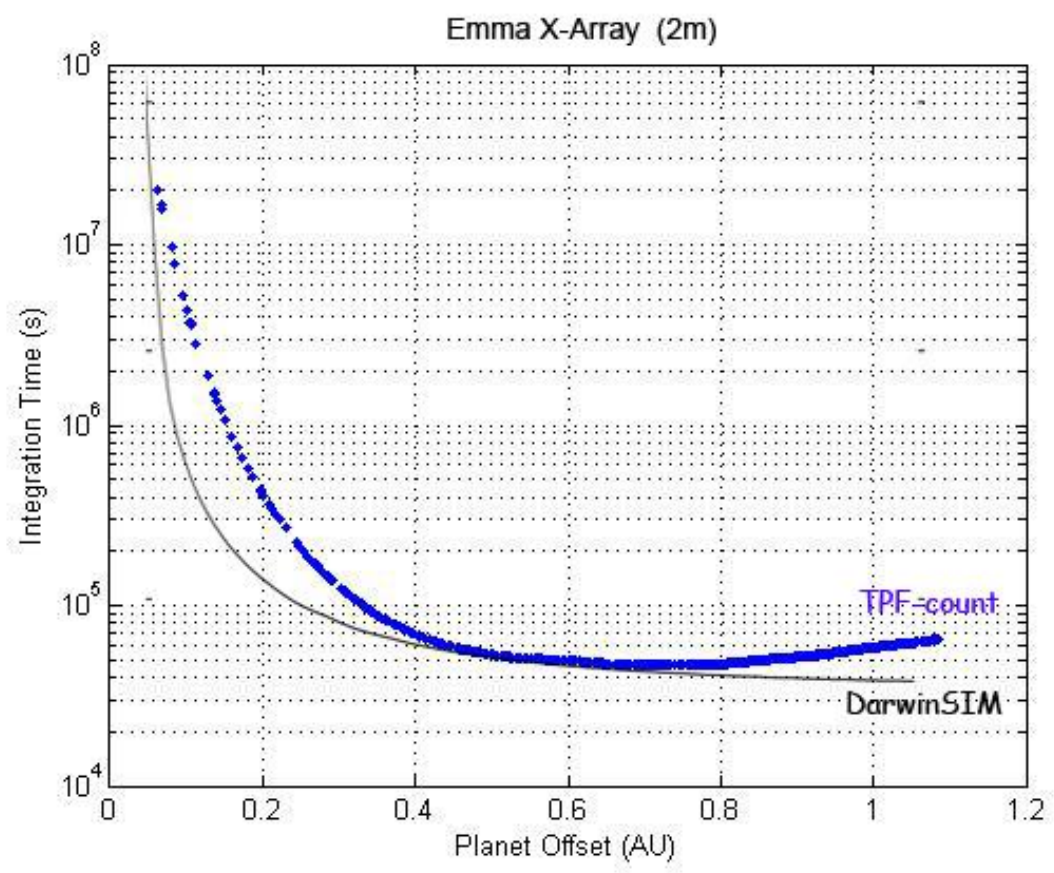

Figure 4. Integration time required to achieve the detection of an Earth-sized habitable planet with respect to the planet offset, computed with DARWINsim and the TPF count model for the Emma X-Array (2-m diameter collectors).

Two types of spectroscopy can be considered: the staring spectroscopy, where the array is kept in a position such that the planet resides on a peak of the modulation map and the rotating spectroscopy, where the array keeps on rotating with respect to the target system so that the planet moves in and out of the peaks of the modulation map. Staring spectroscopy is more efficient in terms of signal acquisition, but requires an accurate knowledge of the planetary orbit. As for the detection phase, the total spectroscopy integration time should not exceed the total time during which the target is visible during the characterization phase. Accounting again for a given fraction of overhead loss, the targets are sorted with respect to ascending integration time, terminated where the cumulative time exceeds the length of nominal characterization period.

Table 1. Parameters adopted for the performance simulations

\begin{tabular}{ll}
\hline \hline Parameter & Emma X-Array Design \\
\hline Collectors & Four spherical mirrors operating at 40 K (nominal aperture diameter: 2 m) \\
Maximum array size & $400 \times 67 \mathrm{~m}$ (aspect ratio 6:1) \\
Wavelength range & $6-20 \mu \mathrm{m}$ \\
Exozodiacal density & Three times our solar system, based on Kelsall model \\
Sky access & Instantaneous $46^{\circ}$ to $83^{\circ}$ from anti-Sun direction \\
Mission duration & 5 years baseline with a goal of 10 years \\
Instrument throughput & $10 \%$ excluding ideal beam combiner losses \\
Planet & Earth-sized planet with a constant temperature of 265 K across the habitable zone \\
Survey time & 2 years \\
Characterization time & 3 years \\
Retargeting time & 6 hours \\
Integration efficiency & $70 \%$ \\
Signal-to-Noise threshold & 5 \\
\hline
\end{tabular}




\section{PERFORMANCE SIMULATION}

\subsection{Modulation efficiency}

As discussed in the introduction, phase chopping is mandatory in order to get rid of the dominant emission of exozodiacal clouds. The principle of phase chopping applied to the X-array configuration is shown in Figure 5 . The outputs of two Bracewell interferometers are combined with opposite phase shifts $( \pm \pi / 2)$ to produce two transmission maps (or "chopped states"). By quickly switching between the two different transmission maps, it is possible to modulate the planet signal without modulating the stellar, local zodiacal cloud, exozodiacal cloud, stray light, thermal, or detector gain contributions to the noise. The chopped response is the so-called modulation map, which contains positive and negative values by construction. Since the value of the modulation map varies across the field-of-view, the position of the planet cannot be unambiguously inferred and an additional level of modulation is mandatory. This is provided by the rotation of the interferometer (typically with a period of 1 day). The two levels of modulation (chopping and rotation) allow to define the "rotational modulation efficiency", which indicates the part of the incoming signal which is actually modulated and thus retrievable by synchronous demodulation. The rotational modulation efficiency for the X-array configuration is about $50 \%$, depending on the radial distance as shown in Figure 6 (right). The left part of Figure 6 show the modulation map in polar coordinates, so that a vertical line would correspond to a complete rotation of the interferometer. Since the planetary position inside the habitable zone is a priori unknown, it is desirable that the effective modulation efficiency is as uniform as possible across the habitable zone to avoid too many reconfigurations (considering different wavelengths). Note that the rotational modulation efficiency for several array configurations has been investigated by Lay. ${ }^{24}$

In order to retrieve the planet signal, the most common approach is correlation mapping, a technique closely related to the Fourier transform used for standard image synthesis. ${ }^{22}$ The result is a correlation map, displayed for a single point source in the lower right part of Figure 5. This represents the Point Spread Function (PSF) of the array. This process, illustrated here for a single wavelength, is repeated across the waveband, and the maps

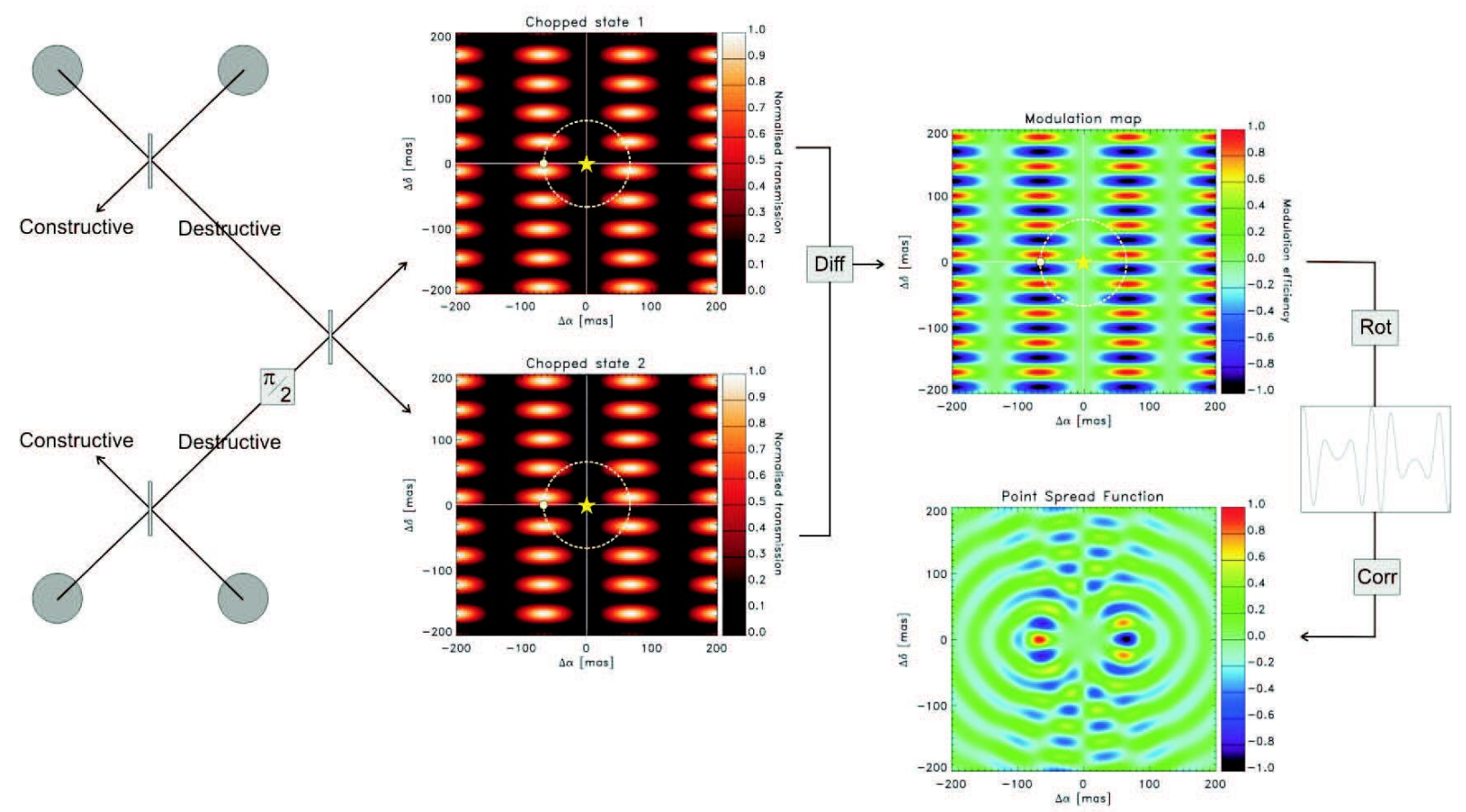

Figure 5. Overview of phase chopping for the X-array configuration, the current baseline for the DARWIN/TPF mission. Combining the beams with different phases produces two conjugated transmission maps (or chopping states), which are used to produce the modulation map. Array rotation then locates the planet by cross-correlation of the modulated chopped signal with a template. 

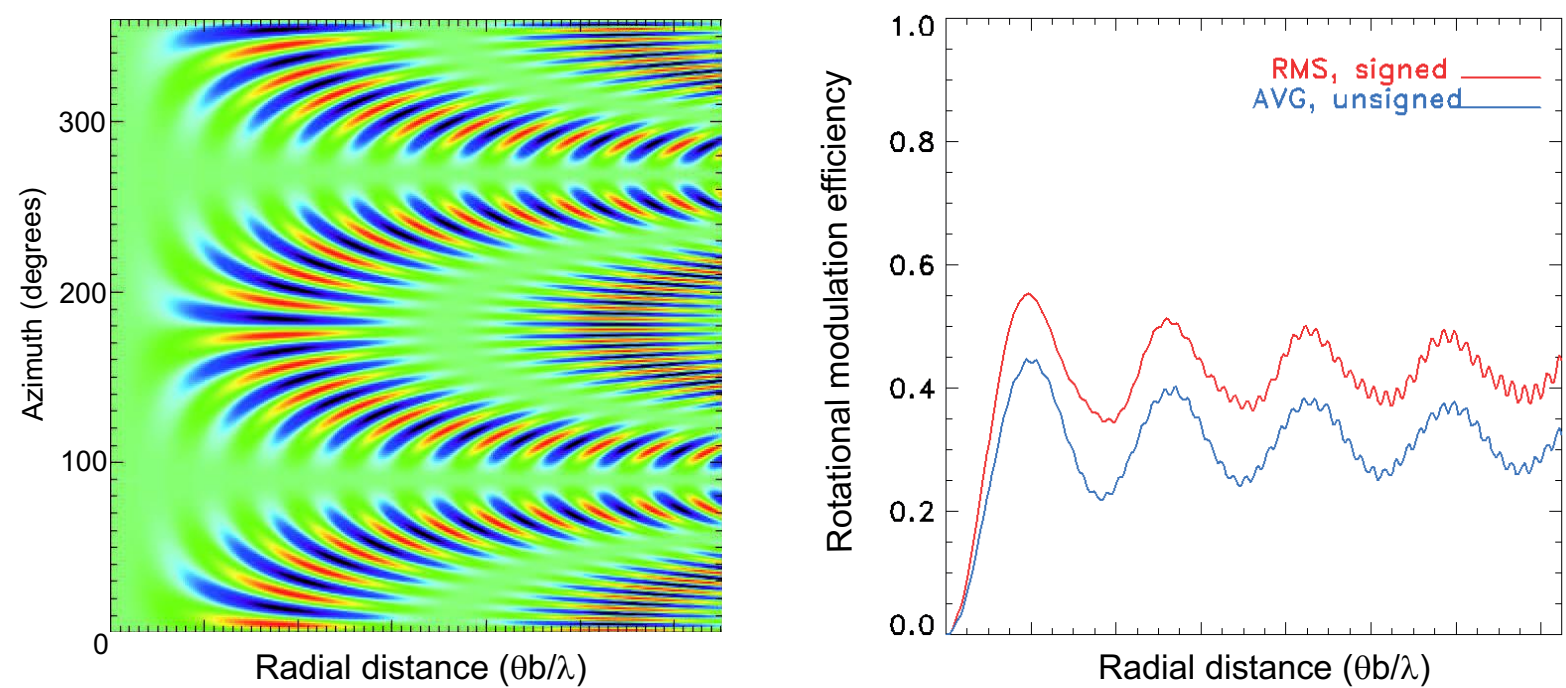

Figure 6. Left, modulation map of the X-array configuration in polar coordinates. Right, corresponding rotational modulation efficiency (red curve, labelled "rms signed"). The blue curve (labelled "avg unsigned") indicates the mean value of the modulation map absolute value.

are co-added to obtain the net correlation map. The broad range of wavelengths planned for DARWIN/TPF greatly extends the spatial frequency coverage of the array, suppressing the side lobes of the PSF.

\subsection{Simulated performance}

As explained in Section 3.1, each simulator first selects from the target catalogue the stars which are observable during one year. In the case of the Emma X-array architecture, 625 nearby main sequence stars are selected from the DARwin catalogue and 1001 from the TPF catalogue. Considering the instrumental parameters listed in Table 1, the results of the simulations for the Emma X-array are presented for different aperture sizes in Table 2. These results have been computed with DARWINsim and validated with the TPF count model by checking that they are consistent within 20\%. The science band has been subdivided into two sub-bands, that match two different fibers: $6-11.5 \mu \mathrm{m}$ and $11.5-20 \mu \mathrm{m}$. In addition, it is assumed that $50 \%$ of the observing time is spent on $\mathrm{G}$ stars, $30 \%$ on $\mathrm{K}$ stars, and $10 \%$ on both $\mathrm{F}$ and $\mathrm{M}$ stars. Each star is assumed to host a terrestrial planet orbiting in the habitable zone. Considering Earth-radius planets within the habitable zone, about 200 stars, well spread among the four selected spectral types, can be surveyed during the nominal 2-year survey. This number reaches about 400 with 3-m aperture telescopes. DARWIN/TPF will thus provide statistically meaningful results on nearby planetary systems. Figure 3 shows that nearby $\mathrm{K}$ and $\mathrm{M}$ dwarfs (small circles to the lower left) are the best-suited targets in terms of Earth-like planet detection capabilities.

For the spectroscopy phase, a required SNR of 5 has been assumed for the detection of $\mathrm{CO}_{2}$ and $\mathrm{O}_{3}$, as discussed in section 3. For the full characterisation (i.e. searching for the presence of $\mathrm{H}_{2} \mathrm{O}, \mathrm{CO}_{2}$, and $\mathrm{O}_{3}$ ), the required SNR has been fixed to 10 on the 7.2-20- $\mu$ m wavelength range. With these assumptions, $\mathrm{CO}_{2}$ and $\mathrm{O}_{3}$ could be searched for about 50 planets (resp. 70) with rotational spectroscopy (resp. staring spectroscopy) while $\mathrm{H}_{2} \mathrm{O}$ could potentially be detected on 24 (resp. 36) planets during the 3-year characterisation phase. These values would be roughly doubled (resp. halved) for 3-m aperture telescopes (resp. 1-m aperture telescopes). Although staring spectroscopy presents (as expected) better results, rotational spectroscopy is more secure since it does not rely on an accurate localisation of the planet. It is also interesting to note that in the case of planets with radii 1.5 time (resp. 2 times) as large as that of Earth, the number of planets for which $\mathrm{H}_{2} \mathrm{O}$ spectroscopy could be performed is doubled (resp. quadrupled), as shown in Table 2. For 2 Earth-radius planets, almost the whole target catalogue (restricted to the part of the sky available to the Emma X-array configuration) could thus be surveyed. 
Table 2. Expected performance in terms of number of stars surveyed and planets characterised during the nominal 5-year mission for various telescope diameters and planet radii (computed with DARWINsim). All stars are assumed to host a planet in the habitable zone and to be surrounded by an exozodiacal cloud 3 times denser that in the solar system.

\begin{tabular}{|c|c|c|c|c|c|}
\hline 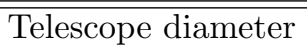 & 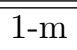 & $2-\mathrm{m}$ & 3-m & $2-\mathrm{m}$ & $2-\mathrm{m}$ \\
\hline Planet radius $\left[R_{\oplus}\right]$ & 1 & 1 & 1 & 1.5 & 2 \\
\hline \multicolumn{6}{|l|}{ Detection } \\
\hline Surveyed ( 5 years) & 119 & 346 & 599 & 611 & 625 \\
\hline Surveyed (2 years) & 76 & 218 & 405 & 437 & 593 \\
\hline$\sharp F$ stars & 5 & 14 & 30 & 36 & 43 \\
\hline$\#$ G stars & 15 & 53 & 100 & 100 & 100 \\
\hline$\sharp \mathrm{K}$ stars & 20 & 74 & 152 & 167 & 241 \\
\hline$\sharp$ M stars & 36 & 77 & 123 & 134 & 209 \\
\hline \multicolumn{6}{|l|}{ Spectroscopy } \\
\hline Staring $\left(\mathrm{CO}_{2}, \mathrm{O}_{3}\right)$ & 26 & 72 & 136 & 173 & 323 \\
\hline Rotating $\left(\mathrm{CO}_{2}, \mathrm{O}_{3}\right)$ & 17 & 49 & 87 & 113 & 217 \\
\hline$\sharp \mathrm{F}$ stars & 1 & 2 & 3 & 6 & 11 \\
\hline$\sharp G$ stars & 4 & 8 & 15 & 22 & 48 \\
\hline$\sharp \mathrm{K}$ stars & 3 & 12 & 25 & 33 & 72 \\
\hline$\sharp$ M stars & 9 & 27 & 44 & 52 & 86 \\
\hline Staring $\left(\mathrm{H}_{2} \mathrm{O}\right)$ & 17 & 36 & 62 & 85 & 170 \\
\hline Rotating $\left(\mathrm{H}_{2} \mathrm{O}\right)$ & 14 & 24 & 43 & 55 & 110 \\
\hline$\sharp$ F stars & 0 & 1 & 1 & 3 & 6 \\
\hline$\sharp \mathrm{G}$ stars & 2 & 4 & 7 & 9 & 21 \\
\hline$\sharp \mathrm{K}$ stars & 1 & 5 & 10 & 14 & 32 \\
\hline$\sharp \mathrm{M}$ stars & 11 & 14 & 25 & 29 & 51 \\
\hline
\end{tabular}

\subsection{Impact of the exozodiacal cloud density}

The amount of warm dust that can be expected around nearby main sequence stars is one of the main design drivers for the DARWIN/TPF missions. Depending on their morphology and brightness, exozodiacal clouds can seriously hamper the capability of a nulling interferometer to detect and characterise habitable terrestrial planets. Considering symmetric face-on exozodiacal discs, Figure 7 shows the SNR variation as a function of the exozodiacal cloud density for different target stars and telescope diameters. We assume that the exozodiacal clouds follow the same density and temperature distribution as in the solar system, ${ }^{27}$ except for a global density scaling factor. To account for this, we introduce the unit zodi, which corresponds to the global dust density in our local zodiacal cloud.

As illustrated in Figure 7, the impact of the exozodiacal cloud density on the SNR is particularly harmful for the hottest target stars which present the brightest exozodiacal discs. For a G2V star located at $10 \mathrm{pc}$, the SNR is reduced by a factor of about 2 between the 3 and 100-zodi cases while for a M0V star located at $10 \mathrm{pc}$ it is only reduced by a factor of about 1.2. Since the local zodiacal cloud emission is generally dominant and obviously the same for all target stars, the distance to the star is also an important parameter. For instance, the SNR is only reduced by a factor of about 1.5 between the 3 and the 100-zodi cases for a G2V star located at $20 \mathrm{pc}$ (vs a factor 2 for a G2V star located at $10 \mathrm{pc}$ ). For larger telescopes, the contribution of the local zodiacal cloud is reduced (thanks to a smaller field-of-view) and the relative impact of the exozodiacal cloud density on the SNR becomes therefore more significant. This is illustrated in Figure 7 for the G2V star located at $10 \mathrm{pc}$ for 2-m and 3-m aperture telescopes. Similar results (although somewhat more pessimistic) have been obtained by Beichman et al. ${ }^{28}$ for a Dual Chopped Bracewell interferometer with 3-m aperture telescopes observing a G2V star located at $10 \mathrm{pc}$. This study has lead to the conclusion that detecting Earth-like planets around star for which the exozodiacal cloud density is larger than 20 zodis would be difficult. However, the present study shows that this is less problematic for smaller aperture telescopes and fainter stars. With 2-m class telescopes, the majority of the DARWIN/TPF target stars can probably tolerate exozodiacal densities as high as 100 zodis, provided that clumps and blobs can be handled by the X-array configuration. Indeed, our simulations assume 


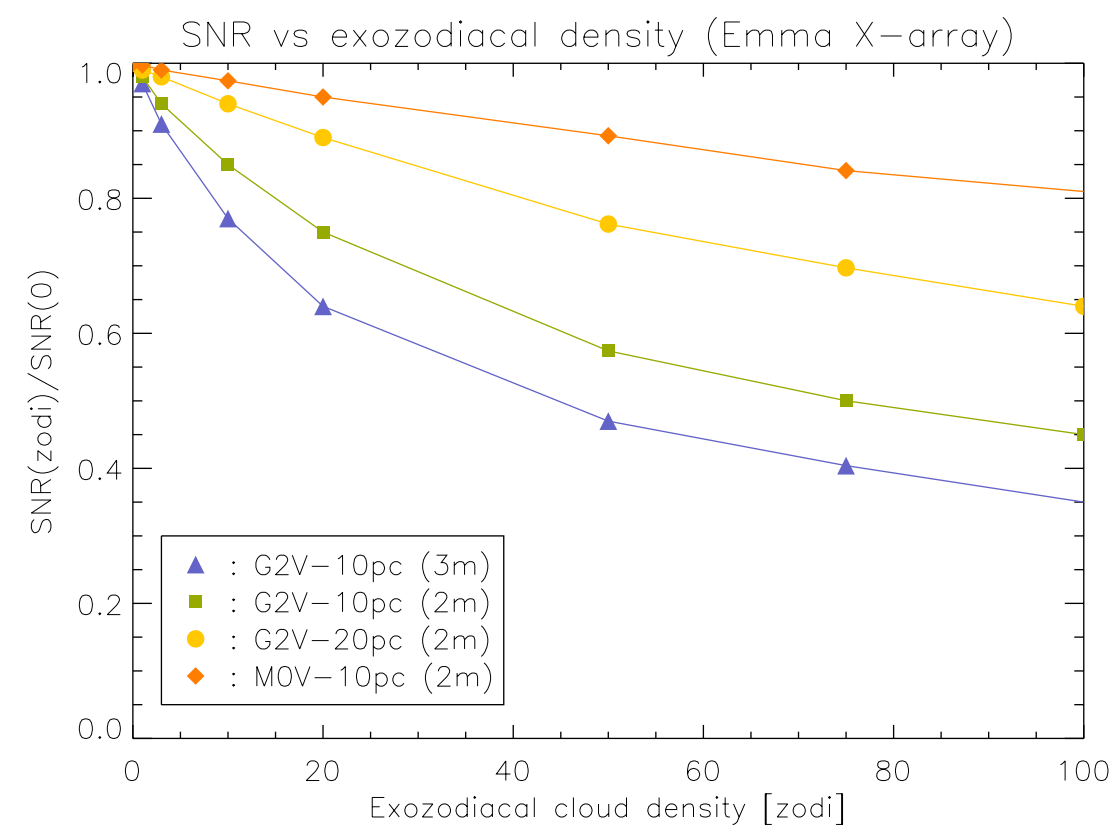

Figure 7. Effect of the exozodiacal cloud density on the SNR for different target stars and telescope diameters. The exozodiacal disc is assumed to follow the Kelsall model ${ }^{27}$ and to be seen in face-on orientation.

that the exozodiacal cloud emission is point-symmetric around the target star, so that it is suppressed by the chopping process (and therefore only contributes to the shot noise). If the exozodiacal cloud is asymmetric or contains significant amounts of clumps and blobs, part of its emission would not be cancelled by the chopping process and could mimic the planetary signal. This behaviour will be particularly harmful for bright exozodiacal clouds. More investigations are necessary to define a secure upper limit on the acceptable exozodiacal cloud density as a function of the spectral type of the target star.

\subsection{Impact of the $\mathrm{H}_{2} \mathrm{O}$ detection strategy}

In this section, we briefly address two different strategies for the $\mathrm{H}_{2} \mathrm{O}$ spectroscopy. As already discussed in section 3, recent results suggest that requiring an SNR of 10 from 7.2 to $20 \mu \mathrm{m}$ would be sufficient for $\mathrm{H}_{2} \mathrm{O}$, $\mathrm{CO}_{2}$, and $\mathrm{O}_{3}$ spectroscopy, provided that the spectral resolution is greater than 20. In particular, this new approach (the A strategy) for $\mathrm{H}_{2} \mathrm{O}$ spectroscopy does not consider the $\mathrm{H}_{2} \mathrm{O}$ band between 6 and $7.2 \mu \mathrm{m}$. On the other hand, the B strategy takes both $\mathrm{H}_{2} \mathrm{O}$ bands into account. The comparative results are displayed in Table 3, showing that requiring an SNR of 10 on the 6-7.2 $\mu \mathrm{m}$ band for $\mathrm{H}_{2} \mathrm{O}$ detection would significantly reduce the efficiency of the spectroscopic phase (the performance is roughly divided by a factor 2). The results of DARWINsim applied to the TPF catalogue are also given for comparison, showing similar results even though the repartition between spectral types is different (due to the reduced amount of M stars in the TPF catalogue).

\section{SUMMARY}

Based on the recent convergence between ESA and NASA on the mission architecture (Emma X-array), we investigate the performance of DARWIN/TPF by using two mission simulators developed independently at ESA/ESTEC and NASA/JPL. For a single target star, the two simulation tools present a very good agreement in SNR, giving similar integration times (with a discrepancy lower than $10 \%$ in average).

With four 2-m diameter collectors, the Emma X-array configuration could detect about 200 Earth-like planets during the 2-year detection phase and perform spectroscopy for about 25 of them in the remaining three years of the mission lifetime. For 2 Earth-radius planets, almost the whole target catalogue could even be surveyed and the number of planets for which $\mathrm{H}_{2} \mathrm{O}$ spectroscopy could be performed reaches about 100. Finally, we 
Table 3. Performance prediction for two $\mathrm{H}_{2} \mathrm{O}$ spectroscopy strategies computed with DARWINsim: the strategy A for which an SNR of 10 is required on the 17.2-20- $\mu \mathrm{m} \mathrm{H}_{2} \mathrm{O}$ band and the strategy B for which an SNR of 10 is also required on the 6.0-7.2- $\mu \mathrm{m}$ band. The results of DARwinsim applied to the TPF catalogue are also given for comparison.

\begin{tabular}{lcc|cc}
\hline \hline Telescope diameter & $2-\mathrm{m}$ & $2-\mathrm{m}$ & $2-\mathrm{m}$ & $2-\mathrm{m}$ \\
Catalogue & DARWIN & TPF & DARWIN & TPF \\
Exozodiacal density & 3 & 3 & 3 & 3 \\
$\mathrm{H}_{2} \mathrm{O}$ strategy & $\mathrm{A}$ & $\mathrm{A}$ & $\mathrm{B}$ & $\mathrm{B}$ \\
\hline Staring $\left(\mathrm{H}_{2} \mathrm{O}\right)$ & 36 & 33 & 21 & 16 \\
Rotating $\left(\mathrm{H}_{2} \mathrm{O}\right)$ & 24 & 19 & 11 & 10 \\
$\sharp \mathrm{F}$ stars & 1 & 1 & 1 & 0 \\
$\sharp \mathrm{G}$ stars & 4 & 5 & 3 & 3 \\
$\sharp \mathrm{K}$ stars & 5 & 10 & 1 & 5 \\
$\sharp \mathrm{M}$ stars & 14 & 3 & 6 & 2 \\
\hline
\end{tabular}

investigate the impact of the exozodiacal cloud density on the mission performance and show that with 2-m aperture telescopes, most DARWIN/TPF target stars can probably tolerate exozodiacal densities as high as 100 zodis, provided that clumps and blobs can be handled by the $\mathrm{X}$-array configuration.

\section{ACKNOWLEDGMENTS}

The authors would like to thank P. Lawson for his support. The first author acknowledges the financial support from the Belgian National Science Foundation ("FRIA").

\section{REFERENCES}

[1] Mayor, M. and Queloz, D., "A Jupiter-Mass Companion to a Solar-Type Star," Nature 378, 355-+ (Nov. 1995).

[2] Udry, S., Bonfils, X., Delfosse, X., Forveille, T., Mayor, M., Perrier, C., Bouchy, F., Lovis, C., Pepe, F., Queloz, D., and Bertaux, J.-L., "The HARPS search for southern extra-solar planets. XI. Super-Earths (5 and $8 \mathrm{M}\{\oplus\})$ in a 3-planet system," $A \& A 4$ 469, L43-L47 (July 2007).

[3] Gillon, M., Pont, F., Demory, B.-O., Mallmann, F., Mayor, M., Mazeh, T., Queloz, D., Shporer, A., Udry, S., and Vuissoz, C., "Detection of transits of the nearby hot Neptune GJ 436 b," A $\dot{G} A$ 472, L13-L16 (Sept. 2007).

[4] Barge, P., Baglin, A., Auvergne, M., Rauer, H., Leger, A., Schneider, J., Pont, F., Aigrain, S., Almenara, J. ., Alonso, R., Barbieri, M., Borde, P., Bouchy, F., Deeg, H. ., De la Reza, R., Deleuil, M., Dvorak, R., Erikson, A., Fridlund, M., Gillon, M., Gondoin, P., Guillot, T., Hatzes, A., Hebrard, G., Jorda, L., Kabath, P., Lammer, H., Llebaria, A., Loeillet, B., Magain, P., Mazeh, T., Moutou, C., Ollivier, M., Patzold, M., Queloz, D., Rouan, D., Shporer, A., and Wuchterl, G., "Transiting exoplanets from the CoRoT space mission I - CoRoT-Exo-1b: a low-density short-period planet around a G0V star," A\&A 482 (Mar. 2008).

[5] Alonso, R., Auvergne, M., Baglin, A., Ollivier, M., Moutou, C., Rouan, D., Deeg, H. J., Aigrain, S., Almenara, J. M., Barbieri, M., Barge, P., Benz, W., Bordé, P., Bouchy, F., De la Reza, R., Deleuil, M., Dvorak, R., Erikson, A., Fridlund, M., Gillon, M., Gondoin, P., Guillot, T., Hatzes, A., Hébrard, G., Kabath, P., Jorda, L., Lammer, H., Léger, A., Llebaria, A., Loeillet, B., Magain, P., Mayor, M., Mazeh, T., Pätzold, M., Pepe, F., Pont, F., Queloz, D., Rauer, H., Shporer, A., Schneider, J., Stecklum, B., Udry, S., and Wuchterl, G., "Transiting exoplanets from the CoRoT space mission II. CoRoT-Exo-2b: A transiting planet around an active G star," A $\mathcal{E} A 482$ (Mar. 2008).

[6] Moutou, C., Pont, F., Barge, P., Aigrain, S., Auvergne, M., Blouin, D., Cautain, R., Erikson, A. R., Guis, V., Guterman, P., Irwin, M., Lanza, A. F., Queloz, D., Rauer, H., Voss, H., and Zucker, S., "Comparative blind test of five planetary transit detection algorithms on realistic synthetic light curves," AESA 437, 355-368 (July 2005). 
[7] Borucki, W. J., Koch, D. G., Lissauer, J., Basri, G., Brown, T., Caldwell, D. A., Jenkins, J. M., Caldwell, J. J., Christensen-Dalsgaard, J., Cochran, W. D., Dunham, E. W., Gautier, T. N., Geary, J. C., Latham, D., Sasselov, D., Gilliland, R. L., Howell, S., Monet, D. G., and Batalha, N., "KEPLER Mission Status," in [Transiting Extrapolar Planets Workshop], Afonso, C., Weldrake, D., and Henning, T., eds., Astronomical Society of the Pacific Conference Series 366, 309-+ (July 2007).

[8] Richardson, L. J., Deming, D., Horning, K., Seager, S., and Harrington, J., "A spectrum of an extrasolar planet," Nature 445, 892-895 (Feb. 2007).

[9] Bracewell, R. N., "Detecting nonsolar planets by spinning infrared interferometer," Nature 274, 780-+ (Aug. 1978).

[10] Angel, J. R. P. and Woolf, N. J., "An Imaging Nulling Interferometer to Study Extrasolar Planets," ApJ 475, 373-+ (Jan. 1997).

[11] Mennesson, B. and Mariotti, J. M., "Array Configurations for a Space Infrared Nulling Interferometer Dedicated to the Search for Earthlike Extrasolar Planets," Icarus 128, 202-212 (July 1997).

[12] Absil, O., Nulling interferometry with IRSI-Darwin: Detection and Characterization of Earth-like Exoplanets, Master's thesis, Liège University, Liège, Belgium, http://www.aeos.ulg.ac.be/ (2001).

[13] Absil, O., Astophysical studies of extrasolar planetary systems using infrared interferometric techniques, $\mathrm{PhD}$ thesis, Liège University, Liège, Belgium (2006).

[14] Fridlund, C. V. M., d'Arcio, L., den Hartog, R., and Karlsson, A., "Status and recent progress of the Darwin mission in the Cosmic Vision program," in [Proc. SPIE], 6268 (July 2006).

[15] Beichman, C., Lawson, P., Lay, O., Ahmed, A., Unwin, S., and Johnston, K., "Status of the terrestrial planet finder interferometer (TPF-I)," in [Proc. SPIE], 6268 (July 2006).

[16] Karlsson, A. L., Wallner, O., Perdigues Armengol, J. M., and Absil, O., "Three telescope nuller based on multibeam injection into single-mode waveguide," in [Proc. SPIE], Traub, W. A., ed., Presented at the Society of Photo-Optical Instrumentation Engineers (SPIE) Conference 5491, 831-+ (Oct. 2004).

[17] Leger, A. and Herbst, T., "DARWIN mission proposal to ESA," ArXiv e-prints 707 (July 2007).

[18] Lay, O. P., "Removing instability noise in nulling interferometers," in [Proc. SPIE], Presented at the Society of Photo-Optical Instrumentation Engineers (SPIE) Conference 6268 (July 2006).

[19] Carle, E., "EMMA configuration: evaluation of optical performances," Tech. Rep. Issue 1, ESA (SCI-A/279) (2005).

[20] den Hartog, R., "The DARWINsim science simulator," Tech. Rep. Issue 1, ESA (SCI-A/297) (May 2005).

[21] Lay, O. P., Martin, S. R., and L., H. S., "Planet-finding performance of the TPF-I Emma architecture," in [Proc. SPIE], 6693 (2007).

[22] Lay, O. P., "Systematic Errors in Nulling Interferometers," Appl. Opt. 43, 6100-6123 (Nov. 2004).

[23] den Hartog, R., "DARWIN science performance prediction," Tech. Rep. Issue 1, ESA (SCI-A/300) (May $2005)$.

[24] Lay, O. P., "Imaging properties of rotating nulling interferometers," Appl. Opt. 44, 5859-5871 (Oct. 2005).

[25] Kaltenegger, L., Eiroa, C., and Fridlund, M., "Target star catalogue for Darwin: Nearby Stellar sample for a search for terrestrial planets," A\& A, submitted (2007).

[26] Fridlund, M., "Darwin Science Requirements Document," Tech. Rep. Issue 5, ESA (SCI-A) (2005).

[27] Kelsall, T., Weiland, J. L., Franz, B. A., Reach, W. T., Arendt, R. G., Dwek, E., Freudenreich, H. T., Hauser, M. G., Moseley, S. H., Odegard, N. P., Silverberg, R. F., and Wright, E. L., "The COBE Diffuse Infrared Background Experiment Search for the Cosmic Infrared Background. II. Model of the Interplanetary Dust Cloud," ApJ 508, 44-73 (Nov. 1998).

[28] Beichman, C. A., Bryden, G., Stapelfeldt, K. R., Gautier, T. N., Grogan, K., Shao, M., Velusamy, T., Lawler, S. M., Blaylock, M., Rieke, G. H., Lunine, J. I., Fischer, D. A., Marcy, G. W., Greaves, J. S., Wyatt, M. C., Holland, W. S., and Dent, W. R. F., "New Debris Disks around Nearby Main-Sequence Stars: Impact on the Direct Detection of Planets," ApJ 652, 1674-1693 (Dec. 2006). 